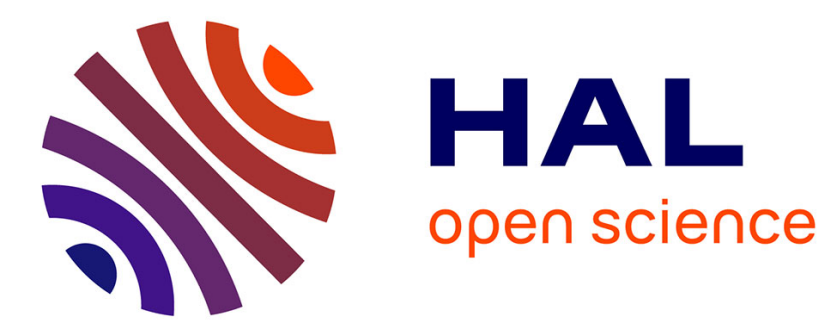

\title{
Macroscopic modeling of double-porosity reservoirs
}

\author{
Pascale Royer, Jean-Louis Auriault, Claude Boutin
}

\section{To cite this version:}

Pascale Royer, Jean-Louis Auriault, Claude Boutin. Macroscopic modeling of double-porosity reservoirs. Journal of Petroleum Science and Engineering, 1996, 16 (4), pp.187 - 202. 10.1016/S09204105(96)00040-X . hal-01713974

\section{HAL Id: hal-01713974 \\ https://hal.science/hal-01713974}

Submitted on 21 Feb 2018

HAL is a multi-disciplinary open access archive for the deposit and dissemination of scientific research documents, whether they are published or not. The documents may come from teaching and research institutions in France or abroad, or from public or private research centers.
L'archive ouverte pluridisciplinaire HAL, est destinée au dépôt et à la diffusion de documents scientifiques de niveau recherche, publiés ou non, émanant des établissements d'enseignement et de recherche français ou étrangers, des laboratoires publics ou privés. 


\title{
Macroscopic modeling of double-porosity reservoirs
}

\author{
P. Royer ${ }^{\mathrm{a}, *}$, J.L. Auriault ${ }^{\mathrm{a}}$, C. Boutin ${ }^{\mathrm{b}}$ \\ a Laboratoire "Sols, Solides, Structures", UJF, INPG, CNRS UMR 5521, BP 53X, F-38041 Grenoble Cedex, France \\ b Laboratoire Géomatériaux, DGCB CNRS URA 1652, École Nationale des Travaux Publics de l'État, rue Maurice Audin, F-69518 Vaux \\ en Velin, France
}

\begin{abstract}
This paper deals with the seepage of a fluid through a fractured porous medium. It summarizes important results obtained using the homogenization method for periodic structures. Thereby, unlike the phenomenological approaches, the macroscopic behaviors are deduced from the physics at the microscopic scales, without any prerequisite. Two cases have been investigated: flow of gas through a rigid medium and flow of incompressible fluid through a deformable matrix. In both situations, it turns out that the ratio between the two separations of scales (macroscopic scale/fissure scale and fissure scale/pore scale) plays an essential role. The macroscopic description depends upon the separations of scales, and the coupling effects between the two porous systems are maximum when the scales are equally separated. Then, the homogenization result is compared to classical phenomenological models for slightly compressible fluid flow through a rigid double-porosity medium. Pseudo-steady-state phenomenological models are shown to give a rough description for transient excitations and finally a correction is proposed giving a more accurate short-time behavior.
\end{abstract}

Keywords: fractured reservoir; double porosity; interporosity flow; physical model

\section{Introduction}

A double-porosity medium consists of two interacting porous systems whose permeabilities are very different. Modeling such an heterogeneous system turms out to be a difficult task. Actually, taking all heterogeneities into account one by one appears to be impossible. Nevertheless, the internal disorder repetition allows a large-scale continuous description. In other words, the physical processes can be described by means of equations with transfer coefficients independent from the macroscopic boundary conditions. This is the basic idea common to all homogenization techniques. If the macroscopic scale is large enough compared to the microscopic scale, a macroscopically equivalent medium can be determined, i.e. behaving "in average" like the initial heteregeneous medium under a given excitation. The homogenization techniques have already proved to be efficient for modeling fluid flows through porous media. The homogenization method for periodic structures leads to precise descriptions since no macroscopic prerequisite is required.

The double-porosity concept was introduced by Barenblatt et al. (1960) for the investigation of fissured 
porous media: one of the two porous structures is associated with the fractures and the other one with the porous matrix. Since many natural reservoirs fit with this scheme, the behavior of such a medium during fluid flow is particularly of interest in mining engineering and petroleum engineering. The first investigations were on the basis of phenomenological approaches, i.e. a directly macroscopic approach (Barenblatt et al., 1960; Barenblatt, 1963; Warren and Root, 1963). The basic assumption of this kind of model is that each point in space is associated with two pressures: the average fluid pressure in the fractures and the average fluid pressure in the pore-matrix. The first model (Barenblatt et al., 1960) shows an important characteristic of a double-porosity system, i.e. the fluid exchange between the two constitutive media. To describe a pseudo-steady-state flow, it is assumed that the interporosity flow occurs in response to the fracture-pore difference in pressure. In the simplified Barenblatt model (Barenblatt, 1963), the flow in the pores as well as the fracture porosity are neglected. As for the Warren and Root model, only the pore-flow is discarded. This kind of simplification is a subject of debate. In particular, let us note the works of Chen $(1989,1990)$, where it is claimed that the overall Barenblatt model should be solved. These three first models - the complete (Barenblatt et al., 1960) and the simplified models (Barenblatt, 1963; Warren and Root, 1963) - have been taken up again by many authors to apply them to different domains of reservoir engineering. A state-of-the-art in the knowledge of double-porosity behavior investigated with this kind of approach is well described in Gringarten (1984), and also in Chen (1989) for the case of slightly compressible fluids.

On the other hand, through the homogenization method for periodic structures, the macroscopic behavior is derived from the complete microscopic description. Now, double-porosity media such as fractured porous media introduce three separated scales, i.e. three scales whose characteristic lengths are very different: the pore scale, the fracture scale and the macroscopic scale. Therefore, the homogenization method for periodic structures is well adapted to model the problem.

The objective of this paper is to summarize important results obtained by this three-scale homogenization approach. The local descriptions at both pore scale and fracture scale are expressed by Navier-Stokes equations. The main result of these investigations is that the macroscopic behavior strongly depends on the relative order of magnitude of the three characteristic lengths. In the next section, the periodic medium under consideration is described, and three cases corresponding to three different relationships between the scale ratios are defined. Section 3, 4 are respectively devoted to gas flow through a rigid fractured porous medium and to incompressible fluid flow through a deformable fractured porous medium. In both sections, attention will be focused on physical interpretation of the results. For details concerning the method and its application, the reader will be referred when required, to several former papers. For a general presentation of the method in the classical case of a two-scale medium, see Auriault (1991). At last in Section 5, a comparison between the results obtained by the phenomenological approach and by homogenization is carried out in the case of slightly compressible fluid flow through a rigid double-porosity medium. It will be shown that the classical pseudo-steady-state model leads to a rough description for transient excitations. This limitation is well notified by the authors of the pseudo-steady-state models (Barenblatt et al., 1990). To be adequate for the purpose of reservoir engineering, these models must be ultimately used in conjunction with well-test data (Warren and Root, 1963). The problem of transient-term introduction in the interporosity-flow term is mentioned in Gringarten (1984) and Chen (1989). Finally, a correction of the interporosity-flow term is proposed, which induces a more accurate short-time description.

\section{Medium under consideration}

\subsection{Medium description}

In order to make the homogenization method for periodic structures consistent with the investigation of three-scale problems, the medium is assumed to be doubly periodic. There is no loss of generality by introducing the assumption of periodicity. It does not have any influence on the macroscopic behavior (Auriault, 


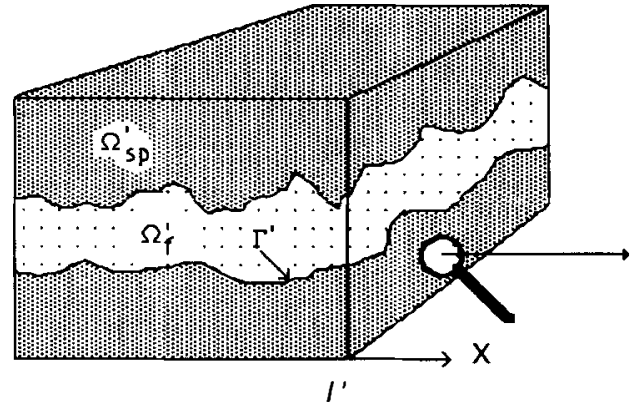

(b)

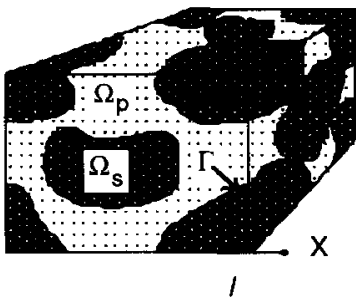

(a)

Fig. 1. a. Pore-scale period.

b. Fracture-scale period.

1991). At the pore scale, consider the medium to be $\Omega$-periodic and its characteristic length to be $l$. The solid and the pores occupy the domains $\Omega_{\mathrm{s}}$ and $\Omega_{\mathrm{p}}$, respectively, and their common boundary is $\Gamma$ (Fig. la). A second periodic porous structure exists at the fracture level, whose period is $\Omega^{\prime}$ and whose characteristic length is $l^{\prime}$, such that $l^{\prime} \gg l$. The porous matrix and the fractures occupy the domains $\Omega_{\mathrm{sp}}^{\prime}$ and $\Omega_{\mathrm{f}}^{\prime}$, respectively, and their common boundary is noted $I^{\prime}$ (Fig. 1b). In a given reservoir $l$ and $l^{\prime}$ are defined, but the macroscopic characteristic length $l^{\prime \prime}$ must be chosen such that: $l^{\prime \prime} \gg l^{\prime}$. In the classical case where only two scales are involved (single-porosity), the homogenization theory can be applied if the two scales are separated, namely, if the macroscopic scale is large enough compared to the microscopic scale. In the case of double porosity, the medium shows two separations of scales:

(1) $l^{\prime} / l^{\prime \prime} \ll 1$ between the fracture scale and the macroscopic level;

(2) $l / l^{\prime} \ll 1$ between the pore scale and the fracture scale.

If the first condition is not checked the homogenization theory cannot be applied because the macroscopic scale and the fracture scale are not separated. If the second one is not checked, that means that the medium is a two-scale medium, namely a single-porosity medium. Thus, we assume here that the two former scale-separation conditions are satisfied, so that the medium is a double-porosity medium and the homogenization method can be applied. Consider the three following parameters picturing the different separations of scales:

$$
\varepsilon=\frac{l}{l^{\prime}}, \quad \eta=\frac{l^{\prime}}{l^{\prime \prime}}, \quad \gamma=\varepsilon \eta=\frac{l}{l^{\prime \prime}}
$$

It will be shown hereafter that the equivalent macroscopic behavior depends upon the relationship between the two separations of scales $\varepsilon$ and $\boldsymbol{\eta}$. In the next subsection three different cases are isolated, which are defined by three different relationships between $\varepsilon$ and $\eta$ resulting from the analysis of characteristic consolidation times of the medium.

\subsection{Characteristic consolidation times of the medium}

Flow of incompressible fluid through a single-porosity deformable medium is governed by the classical model introduced in Biot (1941):

$$
\begin{aligned}
& \vec{\nabla} \cdot(\tilde{C} \tilde{e}-\tilde{\alpha} P)=0 \\
& \vec{\nabla} \cdot(\tilde{K} \tilde{\nabla} P)=\tilde{\alpha} \frac{\partial \tilde{e}}{\partial t}+\beta \frac{\partial P}{\partial t}
\end{aligned}
$$


where $\tilde{C}$ is the material elasticity tensor; $\tilde{e}$ its strain; $\tilde{K}$ its permeability; and $P$ the fluid pressure. Notice the symmetry of Eqs. (1) and (2) set by the tensor $\tilde{\alpha} . \beta>0$ is related to the compressibility of the material of the matrix. $\beta=0$ and $\tilde{\alpha}=\tilde{I}$ when the matrix material is incompressible. Eqs. (1) and (2) are also valid for slightly compressible fluids. The rate of medium strain $(\partial \tilde{e}) /(\partial t)$ is such that:

$$
\left|\frac{\partial \tilde{e}}{\partial t}\right|=O\left(\frac{|\tilde{e}|}{T_{\mathrm{c}}}\right)
$$

where $T_{\mathrm{c}}$ is the characteristic consolidation time of the medium. Let us determine the order of magnitude of $T_{\mathrm{c}}$ with respect to the characteristics of the medium. The tensor $\tilde{\alpha}$ is such that: $|\tilde{\alpha}|=O(1)$. Then, from Eq. (2) and if $L$ is the macroscopic characteristic length of this single-porosity medium, we get:

$$
\left|\frac{\partial \tilde{e}}{\partial t}\right|=O\left(\frac{|\tilde{e}|}{T_{\mathrm{c}}}\right)=O\left(|\tilde{K}| \frac{P}{L^{2}}\right)
$$

Now, from Eq. (1), it follows that:

$$
P=O(|\tilde{C} \| \tilde{e}|)
$$

from which

$$
T_{\mathrm{c}}=O\left(\frac{L^{2}}{|\tilde{K}||\tilde{C}|}\right)
$$

The order of magnitude of the characteristic consolidation time depends upon the physical and geometric characteristics of the material. In a double-porosity medium, two characteristic consolidation times can be defined, $T_{\mathrm{p}}$ for the porous matrix and $T_{\mathrm{f}}$ for the fractures:

$$
\left\{\begin{array}{cc}
\text { (a) In the pores: } & T_{\mathrm{p}}=O\left(\frac{l^{\prime 2}}{\left|\tilde{K}_{\mathrm{p}}\right|\left|\tilde{C}_{\mathrm{p}}\right|}\right) \\
\text { (b) In the fractures: } & T_{\mathrm{f}}=O\left(\frac{l^{\prime 2}}{\left|\tilde{K}_{\mathrm{f}} \| \tilde{C}_{\mathrm{f}}\right|}\right)
\end{array}\right.
$$

The effective elastic coefficients of a porous material with fractures or pores can be assumed to be of the same order of magnitude: $\left|\tilde{C}_{\mathrm{p}}\right|=O\left(\left|\tilde{C}_{\mathrm{f}}\right|\right)$. We have also $\left|\tilde{K}_{\mathrm{p}}\right|=O\left[\left(l^{\prime 2}\right) /(\mu)\right]$ and $\left|\tilde{K}_{\mathrm{f}}\right|=O\left[\left(l^{\prime 2}\right) /(\mu)\right]$, where $\mu$ is the viscosity of the fluid. From these estimations we deduce:

$$
\frac{T_{\mathrm{p}}}{T_{\mathrm{f}}}=O\left(\eta^{2} \varepsilon^{-2}\right)
$$

The ratio between the two characteristic times depends only on the separations of scales. From this last relationship, three cases of interest can be isolated:

\subsubsection{Case I: Weak separation between the pore scale and the fracture scale}

$$
\eta=O\left(\varepsilon^{2}\right)
$$

The small parameter $\varepsilon$ serves as "measurement" and is such that $\varepsilon \ll 1$. It follows:

$$
\frac{T_{\mathrm{p}}}{T_{\mathrm{f}}}=O\left(\varepsilon^{2}\right)
$$


For times of the order of magnitude of $T_{\mathrm{f}}$, the microporous matrix consolidates instantaneously. The coupling between the two porous systems is instantaneous and the characteristic evolution time of the sunken microporous matrix is $T_{\mathrm{f}}$.

\subsubsection{Case II: Equal separations of scales}

$$
\varepsilon=O(\eta)
$$

Hence, the relative order of magnitude of the characteristic consolidation times is such that:

$$
\frac{T_{\mathrm{p}}}{T_{\mathrm{f}}}=O(1)
$$

Phenomena at both pore scale and fracture scale take place at times of the same order of magnitude. Therefore, fractured porous medium consolidation should be strongly influenced by matrix consolidation.

\subsubsection{Case III: Large separation between the pore scale and the fracture scale}

$$
\varepsilon=O\left(\eta^{2}\right)
$$

We get:

$$
\frac{T_{\mathrm{p}}}{T_{\mathrm{f}}}=O\left(\varepsilon^{-1}\right)
$$

Microporous matrix consolidation does not influence fractured medium consolidation. Actually, while the fractures consolidate the matrix behaves like a single-phase elastic medium. Thus, there is no coupling between the two porous systems and the characteristic evolution time in the microporous matrix is $T_{\mathrm{p}}=O\left(\varepsilon^{-1}\right) T_{\mathrm{f}}$.

A similar analysis could be carried out for flow of compressible fluid in a rigid matrix. Eq. (4) remains valid and then the characteristic times $T_{\mathrm{p}}$ and $T_{\mathrm{f}}$ are the flow characteristic times in the pores and in the fractures, respectively.

Hereafter, are successively considered flows of very compressible fluid in a rigid fractured porous medium, and then of incompressible fluid in a deformable fractured porous medium. In each context, the local descriptions in the pores and in the fractures, and the macroscopic behaviors which are derived in the three cases of different scale separation are given.

\section{Gas flow through rigid fractured porous medium}

\subsection{Local descriptions}

Consider quasi-static flow of very compressible fluid in a rigid fractured porous medium. Application of homogenization theory to this problem and calculus are detailed in Royer and Auriault (1992, 1994), Auriault and Royer (1993b), and Royer (1994).

For the sake of simplicity assume the system to be initially at rest: fluid velocity is zero and pressure and density are constant $\left(P_{0}\right.$ and $\rho_{0}$, respectively). Let this equilibrium state be disturbed by changing the boundary conditions at the surface of the porous medium (e.g., by bringing up into service injection or production wells). We assume slow flow, with negligible Reynolds number. The perturbation of the equilibrium state is governed by the following equations, with $k=\mathrm{p}$ in the pores $\left(\Omega_{\mathrm{p}}\right)$ and $k=\mathrm{f}$ in the fractures $\left(\Omega_{\mathrm{f}}^{\prime}\right)$. Fluid pressure and density are $P_{0}+P_{k}$ and $\rho_{0}+\rho_{k}$, respectively, where $P_{k}$ and $\rho_{k}$ are increments. 


\subsubsection{Momentum balance}

Investigation of very compressible fluid flow in a single-porosity deformable porous medium (Auriault et al., 1990) has shown that the inertial and transient terms of Navier-Stokes equations could be neglected for the first-order equivalent macroscopic description. This result is still valid in the context of a double-porosity medium. Thus, the momentum balance is written as follows:

$$
\mu \Delta \vec{v}_{k}+(\lambda+\mu) \vec{\nabla}\left(\vec{\nabla} \cdot \vec{v}_{k}\right)-\vec{\nabla} P_{k}=\overrightarrow{0}
$$

where $\lambda$ and $\mu$ are viscosities; $\vec{v}_{k}$ the velocity; and $P_{k}$ the pressure increment.

\subsubsection{Mass balance}

The mass balance is expressed by:

$$
\frac{\partial \rho_{k}}{\gamma_{t}}+\vec{\nabla} \cdot\left[\left(\rho_{0}+\rho_{k}\right) \vec{v}_{k}\right]=0
$$

where $\rho_{0}$ is the initial density; and $\rho_{k}$ the density increment.

\subsubsection{Gas equation of state}

For the sake of simplicity, assume the fluid to be linear. Then:

$$
\rho_{k}=\frac{\rho_{0}}{P_{0}} P_{k}
$$

where $\rho_{0} / P_{0}=A$ is a constant.

A more realistic equation of state can be easily introduced.

\subsubsection{Boundary conditions}

3.1.4.1. On $\Gamma$. Classically, the adherence condition is:

$$
\vec{v}_{\mathrm{p}} / \Gamma=\overrightarrow{0}
$$

3.1.4.2. On $\Gamma^{\prime}$. Continuity of normal flux and pressure are written as:

$$
\begin{aligned}
& \vec{v}_{\mathrm{f}} / \Gamma^{\prime} \cdot \vec{N}^{\prime}=\left\langle\vec{v}_{\mathrm{p}}\right\rangle_{\Omega} \cdot \vec{N}^{\prime}=\frac{1}{|\Omega|} \int_{\Omega_{\mathrm{p}}} \vec{v}_{\mathrm{p}} \mathrm{d} \Omega \cdot \vec{N}^{\prime} \\
& P_{\mathrm{f}}=P_{\mathrm{p}}
\end{aligned}
$$

$\overrightarrow{N^{\prime}}$ is a unit and normal to $\Gamma^{\prime}$ vector. It has been shown that the volume average $\left\langle\vec{v}_{\mathrm{p}}\right\rangle_{\Omega}$ is equal to a surface average (Levy and Sanchez-Palencia, 1975). Therefore, the velocity $\overrightarrow{\vec{v}}_{\mathrm{f}} /_{\Gamma}^{\prime}$ is well defined by Eq. (9).

\subsubsection{Initial conditions}

The fluid is at rest at the initial time:

$$
\begin{aligned}
& \vec{v}_{k}(t=0)=\overrightarrow{0} \\
& \rho_{k}(t=0)=0 \\
& P_{k}(t=0)=0
\end{aligned}
$$

The above set of equations constitutes the local descriptions in the pores and in the fractures on which the homogenization is then applied to derive the three macroscopic behaviors. The differences between the three 
cases in the local descriptions is highlighted when this set of equations is cast in dimensionless form. For details concerning the method, the reader is referred to Royer (1994) and Royer and Auriault (1994).

\subsection{Macroscopic descriptions}

\subsubsection{Macroscopic description in case I}

Case I has been defined by the following relationship between the separations of scales:

$$
\eta=O\left(\varepsilon^{2}\right), \quad \varepsilon \ll 1
$$

The observer located at the macroscopic scale sees the pores and the fractures as being nearly of the same size. In this case, the derived macroscopic behavior for times of the order of magnitude of $T_{\mathrm{f}}$ is:

$$
\left[\phi^{\prime}+\left(1-\phi^{\prime}\right) \phi\right] \frac{\partial P}{\partial t}-\vec{\nabla} \cdot\left[\left(P_{0}+P\right) \tilde{K}_{f} \vec{\nabla} P\right]=0
$$

and the macroscopic velocity is:

$$
\vec{V}=-\tilde{K}_{\mathrm{f}} \vec{\nabla} P
$$

where $\tilde{K}_{\mathrm{f}}$ is the fracture permeability; $P_{0}$ is the initial pressure; $P=P_{\mathrm{p}}=P_{\mathrm{f}}$ (the pressure does not depend on the local space variable on $\Omega_{\mathrm{sp}}^{\prime}$ and $\Omega_{\mathrm{f}}^{\prime} ; \phi=\left|\Omega_{\mathrm{p}}\right| /|\Omega|$ and $\phi^{\prime}=\left|\Omega_{\mathrm{f}}^{\prime}\right| /\left|\Omega^{\prime}\right|$ are the porosities of the pores and of the fractures, respectively; and $\phi+\left(1-\phi^{\prime}\right) \phi$ is the total porosity.

This macroscopic behavior is a non-linear mass-balance equation with a classical Darcy's law in the fractures. Flow in the pores is instantaneous. The porous matrix plays the role of a fluid reservoir as it is shown through the source term $\left(\phi+\left(1-\phi^{\prime}\right) \phi\right)(\partial P / \partial t)$. Actually, in the single-porosity model, this source term is simply $\phi^{\prime}(\partial P / \partial t)$. Here, the porosity of the whole volume appears.

\subsubsection{Macroscopic description in case II}

Case II is the case where the separations of scales are of the same order of magnitude:

$$
\varepsilon=O(\eta), \varepsilon \ll 1
$$

This is the case for which the most important coupling effect between both flows is obtained. This result was foreseeable from the investigation of the characteristic consolidation times: $T_{\mathrm{p}}=O\left(T_{\mathrm{f}}\right)$. For times $O\left(T_{\mathrm{f}}\right)=O\left(T_{\mathrm{p}}\right)$, the derived macroscopic behavior is:

$$
\phi^{\prime} \frac{\partial P_{\mathrm{f}}}{\partial t}+\phi \frac{\partial\left\langle P_{\mathrm{p}}\right\rangle_{\text {eff }}}{\partial t}-\vec{\nabla} \cdot\left[\left(P_{0}+P_{\mathrm{f}}\right) \tilde{K}_{\mathrm{f}} \vec{\nabla} P_{\mathrm{f}}\right]=0
$$

where the notations are the same as in case I, and where

$$
\left\{\begin{array}{l}
\text { (1) } P_{\mathrm{p}} \text { is defined by the following boundary }- \text { value problem on } \Omega^{\prime}: \\
\text { (a) } \phi \frac{\partial P_{\mathrm{p}}}{\partial t}-\vec{\nabla} \cdot\left[\left(P_{0}+P_{\mathrm{p}}\right) \tilde{K_{\mathrm{p}}} \vec{\nabla} P_{\mathrm{p}}\right]=0 \\
\text { (b) } P_{\mathrm{p}}=P_{\mathrm{f}} \text { on } \Gamma^{\prime} \\
\text { where } P_{\mathrm{p}} \text { is } \Omega^{\prime}-\text { periodic } \\
\text { (2) }\left\langle P_{\mathrm{p}}\right\rangle_{\text {eff }}=\frac{1}{\left|\Omega^{\prime}\right|} \int_{\Omega_{\mathrm{sp}}^{\prime}} P_{\mathrm{p}} \mathrm{d} \Omega
\end{array}\right.
$$


The macroscopic velocity is:

$$
\vec{V}=-\tilde{K}_{\mathrm{f}} \vec{\nabla} P_{\mathrm{f}}
$$

It turns out that: $P_{\mathrm{p}}=\mathscr{F}\left(P_{\mathrm{f}}\right)$, where $\mathscr{F}$ is a nonlinear time-dependent functional exhibiting memory effects.

The macroscopic behavior is strongly influenced by flow in the pores. It induces memory effects and strong non-linearities. As it highlights how the local effects may affect the macroscopic description, this case is the most interesting one.

\subsubsection{Macroscopic description in case III}

Case III is defined by the following relationship:

$$
\varepsilon=O\left(\eta^{2}\right), \quad \varepsilon \ll 1
$$

Here, the observer located at the macroscopic scale sees the pores and the fractures as being of very different sizes. For times $O\left(T_{\mathrm{f}}\right)$, the macroscopic description is:

$$
\phi^{\prime} \frac{\partial P_{\mathrm{f}}}{\partial t}-\vec{\nabla} \cdot\left[\left(P_{0}+P_{\mathrm{f}}\right) \tilde{K}_{\mathrm{f}} \vec{\nabla} P_{\mathrm{f}}\right]=0
$$

and the macroscopic velocity is:

$$
\vec{V}=-\tilde{K_{\mathrm{f}}} \vec{\nabla} P_{\mathrm{f}}
$$

The macroscopic description is derived without the need of the behavior in the pores. There is no coupling effect between phenomena at the first order of approximation. The result describes the behavior of a single-porosity medium, that only consists of the fracture porous system. The flow in the pores is completely ignored at the first order of approximation.

\subsection{Concluding remarks}

The strongest coupling effects are obtained in case II, i.e. for equally separated scales. The models obtained in case I and case III can, afterwards, be deduced from the case II model. The reasoning is based on the boundary-value problem defining $P_{\mathrm{p}}$ in the case II model:

$$
\left\{\begin{array}{l}
\text { (a) } \phi \frac{\partial P_{\mathrm{p}}}{\partial T}-\vec{\nabla} \cdot\left[\left(P_{0}+P_{\mathrm{p}}\right) \tilde{K_{\mathrm{p}}} \vec{\nabla} P_{\mathrm{p}}\right]=0 \\
\text { (b) } P_{\mathrm{p}}=P_{\mathrm{f}} \text { on } \Gamma^{\prime} \\
\text { where } P_{\mathrm{p}} \text { is } \Omega^{\prime} \text { - periodic }
\end{array}\right.
$$

Since $P_{\mathrm{p}}=O\left(P_{0}\right)$, the following "consolidation" characteristic length can be defined:

$$
L_{\mathrm{c}}=\left(P_{0} K_{\mathrm{p}} T_{\mathrm{f}} / \phi\right)^{1 / 2}
$$

In case I we have $L_{\mathrm{c}} \gg l^{\prime}$, then $P_{\mathrm{p}} \rightarrow P_{\mathrm{f}}$ and Eq. (15) becomes Eq. (14). In case III we have $L_{\mathrm{c}} \ll l^{\prime}$, then only a negligible part of the period $\Omega^{\prime}$ is affected by the consolidation process. Therefore, almost all the volume remains at rest and $P_{\mathrm{p}}=0$ on average. Thus, Eq. (15) becomes Eq. (16). 


\section{Incompressible fluid flow through deformable fractured porous medium}

\subsection{Local descriptions}

Consider now quasi-static flow of incompressible fluid in a deformable fractured porous medium. This context is a part of the general theory regarding fluid motion in deformable porous media, also called consolidation. Hereafter, results obtained in Auriault and Boutin (1992, 1993), and Boutin (1994) are taken up again.

Consolidation description of a medium under fluid action requires solid deformation as well as fluid flow knowledge. As previously, let us assume the system to be at rest at the initial time: the velocity is zero-valued and the pressure is constant and $P_{0}$-valued.

\subsubsection{Solid matrix quasi-static momentum balance}

Matrix deformability is governed by the classical law of infinitesimal linear elasticity:

$$
\vec{\nabla} \tilde{\sigma}_{\mathrm{s}}=\overrightarrow{0}
$$

with

$$
\tilde{\sigma_{\mathrm{s}}}=\tilde{a} \tilde{e}\left(\vec{u}_{\mathrm{s}}\right)
$$

where $\tilde{\sigma}_{\mathrm{s}}$ is the solid matrix stress tensor; $\tilde{e}$ the strain tensor; $\tilde{a}$ the elastic tensor; and $\vec{u}_{\mathrm{s}}$ the solid matrix displacement field.

\subsubsection{Fluid momentum balance}

As previously, it is written as:

$$
\mu \Delta \vec{v}_{k}-\vec{\nabla} P_{k}=\overrightarrow{0}
$$

which can also be expressed by:

$$
\vec{\nabla} \tilde{\boldsymbol{\sigma}}_{k}=\overrightarrow{0}
$$

with

$$
\tilde{\sigma}_{k}=-P_{k} \tilde{I}+2 \mu \tilde{D}
$$

and where

$$
\tilde{D}=\tilde{e}\left(\vec{v}_{k}\right)
$$

is the rate of fluid strain tensor.

\subsubsection{Mass balance}

In the case of incompressible fluid it is written as:

$$
\vec{\nabla} \cdot \vec{v}_{k}=0
$$

\subsubsection{Boundary conditions}

4.1.4.1. On $\Gamma$. Continuity of normal stress and velocity are written:

$$
\tilde{\sigma}_{\mathrm{s}} \vec{N}=\tilde{\sigma}_{\mathrm{p}} \vec{N}
$$


where $\vec{N}$ is a unit and normal to $I$ vector,

$$
\vec{v}_{\mathrm{s}}=\vec{v}_{\mathrm{p}}
$$

4.1.4.2. On $\Gamma^{\prime}$. Continuity of normal total stress is expressed by:

$$
\left(\left\langle\tilde{\sigma}_{\mathrm{s}}\right\rangle_{\Omega}+\left\langle\tilde{\sigma}_{\mathrm{p}}\right\rangle_{\Omega}\right) \vec{N}^{\prime}=\tilde{\sigma}_{\mathrm{f}} \vec{N}^{\prime}
$$

where $\vec{N}^{\prime}$ is a unit and normal to $\Gamma^{\prime}$ vector.

Continuity of solid relative fluid velocity and of pressure are given by:

$$
\begin{aligned}
& \left(\vec{v}_{\mathrm{f}}-\left\langle\vec{v}_{\mathrm{s}}\right\rangle_{\Omega}\right) \cdot \vec{N}^{\prime}=\left\langle\vec{v}_{\mathrm{p}}\right\rangle_{\Omega} \cdot \vec{N}^{\prime} \\
& P_{\mathrm{p}}=P_{\mathrm{f}}
\end{aligned}
$$

\subsection{Macroscopic descriptions}

\subsubsection{Macroscopic description in case 1}

In case I, the pores and the fractures are of nearly the same sizes when they are observed from the macroscopic scale. For times $O\left(T_{\mathrm{f}}\right)$, the macroscopic behavior of incompressible fluid flow through a deformable fractured porous matrix is written:

$$
\begin{aligned}
& \vec{\nabla}\left[\tilde{C} \tilde{e}\left(\vec{u}_{\mathrm{s}}\right)-\tilde{\alpha} P\right]=0 \\
& \tilde{\nabla} \cdot\left(\tilde{K_{\mathrm{f}}} \vec{\nabla} P\right)=\tilde{\gamma} \tilde{e}\left(\frac{\partial \vec{u}_{\mathrm{s}}}{\partial t}\right)+\beta \frac{\partial P}{\partial t}, \quad \tilde{\alpha} \neq \tilde{\gamma}
\end{aligned}
$$

and the macroscopic fluid velocity is:

$$
\begin{aligned}
& \vec{V}=-\tilde{K}_{\mathrm{f}} \vec{\nabla} P \\
& P=P_{\mathrm{p}}=P_{\mathrm{f}}
\end{aligned}
$$

Note that for a single-porosity medium the equations are similar but with equal $\tilde{\alpha}$ and $\tilde{\gamma}$ tensors (see Eqs. (1) and (2) for comparison). Thus, the secondary porosity induces the break of this symmetry.

\subsubsection{Macroscopic description in case II}

In case II, i.e. for equal separations of scales, the macroscopic behavior at constant pulsation and for times $O\left(T_{\mathrm{f}}\right)=O\left(T_{\mathrm{p}}\right)$ is the following:

$$
\begin{aligned}
& \vec{\nabla}\left[\tilde{C} \tilde{e}\left(\vec{u}_{\mathrm{s}}\right)-\tilde{\alpha} P_{\mathrm{f}}\right]=0 \\
& \vec{\nabla} \cdot\left(\tilde{K_{\mathrm{f}}} \vec{\nabla} P_{\mathrm{f}}\right)=\tilde{\gamma} \tilde{e}\left(\frac{\partial \vec{u}_{\mathrm{s}}}{\partial t}\right)+\beta \frac{\partial P_{\mathrm{f}}}{\partial t}, \quad \tilde{\alpha} \neq \tilde{\gamma}
\end{aligned}
$$

and the macroscopic fluid velocity is:

$$
\vec{V}=-\tilde{K_{\mathrm{f}}} \vec{\nabla} P_{\mathrm{f}}
$$


The pore pressure $P_{\mathrm{p}}$ is defined by the following boundary-value problem on the fracture periodic cell:

$$
\left\{\begin{array}{l}
\text { (a) } \vec{\nabla}_{y} \cdot\left(\tilde{K}_{\mathrm{p}} \vec{\nabla}_{y} P_{\mathrm{p}}\right)=\tilde{\gamma}_{\mathrm{p}}\left[\tilde{e}_{y}\left(\vec{u}_{\mathrm{s}}^{1}\right)+\tilde{e}\left(\vec{u}_{\mathrm{s}}^{0}\right)\right]+\beta_{\mathrm{p}} \frac{\partial P_{\mathrm{p}}}{\partial t} \\
\text { (b) } P_{\mathrm{p}}=P_{\mathrm{f}} \text { on } \Gamma^{\prime} \\
P_{\mathrm{p}} \text { is } \Omega^{\prime}-\text { periodic }
\end{array}\right.
$$

where $\vec{y}$ is the fracture-scale space variable; and $\tilde{e}_{y}$ and $\vec{\nabla}_{y}$ are the strain tensor and the nabla operator expressed with the fracture-scale space variable, respectively. $\overrightarrow{\boldsymbol{u}}_{\mathrm{s}}^{0}$ and $\overrightarrow{\boldsymbol{u}}_{\mathrm{s}}^{1}$ are the two first terms of the displacement asymptotic expansion [see Auriault (1991) for a general introduction of the homogenization method and Auriault and Boutin (1993) for details about case II results].

As in case I, the coupling tensors symmetry is broken. But unlike the first case, the pore pressure is different from the fracture pressure: $P_{\mathrm{p}}$ appears as a linear function of $\tilde{e}\left(\vec{u}_{\mathrm{s}}\right)$ and $P_{\mathrm{f}}$. On the other side, the tensors $\tilde{C}, \tilde{\gamma}$ and $\tilde{\alpha}$ and the scalar $\beta$ are now complex-valued and pulsation-dependent in Fourier space. Therefore, these quantities show memory effects for transient excitations, i.e. they are expressed with convolution products. Thus, the behavior at a given time will depend on the behavior during the previous time interval.

\subsubsection{Macroscopic description in case III}

In case III, i.e. for pores and fractures of very different sizes, the macroscopic behavior for times $O\left(T_{\mathrm{f}}\right)$ is:

$$
\begin{aligned}
& \vec{\nabla}\left[\tilde{C} \tilde{e}\left(\tilde{u}_{\mathrm{s}}\right)-\tilde{\alpha} P_{\mathrm{f}}\right]=0 \\
& \vec{\nabla} \cdot\left(\tilde{K_{\mathrm{f}}} \vec{\nabla} P_{\mathrm{f}}\right)=\tilde{\gamma} \tilde{e}\left(\frac{\partial \vec{u}_{\mathrm{s}}}{\partial t}\right)+\beta \frac{\partial P_{\mathrm{f}}}{\partial t}, \quad \tilde{\alpha}=\tilde{\gamma}
\end{aligned}
$$

and the macroscopic fluid velocity is:

$$
\vec{V}=-\tilde{K}_{\mathrm{f}} \vec{\nabla} P_{\mathrm{f}}
$$

This is a single-porosity medium behavior, with a coupling symmetry. Flow in the pores is completely ignored at the first order of approximation. Only the pressure in the fractures is required for the description.

\section{Comparison between phenomenological and homogenization models}

\subsection{Introduction}

To our knowledge, there is no rigorous phenomenological model for highly compressible fluid flow in the literature. The existing rigorous models are derived for slightly compressible fluids. To describe a pseudosteady-state flow, it is assumed that the interporosity flow $q$, i.e. the flux of fluid from matrix to fractures, occurs in response to the fracture-pore difference in pressure:

$$
q=s K_{\mathrm{p}}\left(P_{\mathrm{p}}-P_{\mathrm{f}}\right)
$$

where $s$ is a characteristic coefficient of the fractured rock proportional to the specific surface of the block.

In this section the different phenomenological models are first introduced. Then, one of them, namely, the Warren and Root model is compared to the case II model when $t$ goes to infinity, but in a linearized form and for a slightly compressible fluid flow through a rigid double-porosity matrix. This comparison will allow us to improve the interporosity term. 


\subsection{Complete Barenblatt model}

To describe a double-porosity model, Barenblatt et al. (1960) introduced two pressure fields at each point of space: $P_{\mathrm{f}}$ and $P_{\mathrm{p}}$ for the fractures and the pores, respectively. The model applies to the case of a slightly deformable matrix and a slightly compressible fluid. When the porous matrix is rigid, the model reduces to the two following equations:

$$
K_{\mathrm{f}} \Delta P_{\mathrm{f}}=\phi^{\prime} C^{*} \frac{\partial P_{\mathrm{f}}}{\partial t}-s K_{\mathrm{p}}\left(P_{\mathrm{p}}-P_{\mathrm{f}}\right)
$$

and

$$
K_{\mathrm{p}} \Delta P_{\mathrm{p}}=\phi C^{*} \frac{\partial P_{\mathrm{p}}}{\partial t}+s K_{\mathrm{p}}\left(P_{\mathrm{p}}-P_{\mathrm{f}}\right)
$$

where $C^{*}$ is the gas compressibility coefficient. The permeability tensors are assumed to be isotropic: $\tilde{K}_{\mathrm{f}}=K_{\mathrm{f}} \tilde{I}, \tilde{K}_{\mathrm{p}}=K_{\mathrm{p}} \tilde{I}$, where $\tilde{I}$ is the identity tensor.

\subsection{Simplified Barenblatt model}

In the case of fractured porous medium, the Barenblatt theory neglects the fracture porosity $\phi^{\prime}$ and the fluid flow in the porous matrix (Barenblatt, 1963). Consequently, the pore permeability only takes place to describe interporosity flow, i.e. the fluid exchange between the pore matrix and the fractures:

$$
\begin{aligned}
& K_{\mathrm{f}} \Delta P_{\mathrm{f}}+s K_{\mathrm{p}}\left(P_{\mathrm{p}}-P_{\mathrm{f}}\right)=0 \\
& \phi C^{*} \frac{\partial P_{\mathrm{p}}}{\partial t}+s K_{\mathrm{p}}\left(P_{\mathrm{p}}-P_{\mathrm{f}}\right)=0
\end{aligned}
$$

Elimination of $P_{\mathrm{p}}$ in Eqs. (37) and (38) yields:

$$
\frac{\partial P_{\mathrm{f}}}{\partial t}-\frac{K_{\mathrm{f}}}{s K_{\mathrm{p}}} \frac{\partial}{\partial t}\left(\Delta P_{\mathrm{f}}\right)-\frac{K_{\mathrm{f}}}{\phi C^{*}} \Delta P_{\mathrm{f}}=0
$$

\subsection{Warren and Root model}

The double-porosity model of Warren and Root (1963) is also a simplification of the complete Barenblatt model. The porosity and the compressibility coefficient in the fractures are not neglected, but fluid flow in the pore matrix is neglected in this model:

$$
\begin{aligned}
& K_{\mathrm{f}} \Delta P_{\mathrm{f}}=\phi^{\prime} C^{*} \frac{\partial P_{\mathrm{f}}}{\partial t}-s K_{\mathrm{p}}\left(P_{\mathrm{p}}-P_{\mathrm{f}}\right) \\
& \phi C^{*} \frac{\partial P_{\mathrm{p}}}{\partial t}+s K_{\mathrm{p}}\left(P_{\mathrm{p}}-P_{\mathrm{f}}\right)=0
\end{aligned}
$$

In Fourier space, the system is written as:

$$
\begin{aligned}
& K_{\mathrm{f}} \Delta P_{\mathrm{f}}=\mathrm{i} \omega \phi^{\prime} C^{*} P_{\mathrm{f}}-s K_{\mathrm{p}}\left(P_{\mathrm{p}}-P_{\mathrm{f}}\right) \\
& \text { i } \omega \phi C^{*} P_{\mathrm{p}}+s K_{\mathrm{p}}\left(P_{\mathrm{p}}-P_{\mathrm{f}}\right)=0
\end{aligned}
$$

where $\omega$ is the pulsation. 
Elimination of $P_{\mathrm{p}}$ between Eqs. (42) and (43) yields:

$$
K_{\mathrm{f}} \Delta P_{\mathrm{f}}=\mathrm{i} \omega \phi^{\prime} C^{*} P_{\mathrm{f}}+\frac{\mathrm{i} \omega \phi C^{*} s K_{\mathrm{p}}}{s K_{\mathrm{p}}+\mathrm{i} \omega \phi C^{*}} P_{\mathrm{f}}
$$

\subsection{Linearized model obtained by homogenization}

For the comparison to be possible, the case II model obtained in Section 3 is rewritten for slightly compressible fluids. The equation of state (7) is replaced by:

$$
\rho_{0}+\rho_{k}=\rho_{0}\left(1+C^{*} P_{k}\right), \quad k=\mathrm{p}, \mathrm{f}
$$

where $\left|C^{*} P_{k}\right| \ll 1$; and $C^{*}$ is the compressibility coefficient. We assume the permeabilities to be constant and isotropic. Then, it is possible to show that the model is changed to:

$$
\phi^{\prime} C^{*} \frac{\partial P_{\mathrm{f}}}{\partial t}+\phi C^{*} \frac{\partial\left\langle P_{\mathrm{p}}\right\rangle_{\mathrm{eff}}}{\partial t}-K_{\mathrm{f}} \Delta P_{\mathrm{f}}=0
$$

$P_{\mathrm{p}}$ is the solution of the following diffusive equation:

$$
K_{\mathrm{p}} \Delta_{y} P_{\mathrm{p}}=\phi C^{*} \frac{\partial P_{\mathrm{p}}}{\partial t}
$$

with the boundary condition

$$
P_{\mathrm{p}}=P_{\mathrm{f}} \text { on } \Gamma^{\prime}
$$

where $\vec{y}$ is the fracture-scale space variable and $\Delta_{y}$ the Laplace operator expressed with the fracture-scale space variable.

Proceeding by Fourier analysis, we then have to solve:

$$
K_{\mathrm{p}} \Delta_{y} P_{\mathrm{p}}=\phi C^{*} \text { i } \omega P_{\mathrm{p}}, \quad P_{\mathrm{p}}=P_{\mathrm{f}} \text { on } \Gamma^{\prime}
$$

Let us define $W$ by:

$$
P_{\mathrm{p}}=P_{\mathrm{f}}+W
$$

The boundary-value problem becomes:

$$
K_{\mathrm{p}} \Delta_{y} W=\phi C^{*} \mathrm{i} \omega\left(P_{\mathrm{f}}+W\right), \quad W=0 \text { on } \Gamma^{\prime}
$$

The solution is linear with respect to $P_{\mathrm{f}}$ :

$$
W(\vec{y}, \vec{x}, \omega)=-k(\vec{y}, \vec{x}, \omega) P_{\mathrm{f}}
$$

where $\vec{x}$ is the macroscopic-scale space variable; and $k$ is complex-valued and $\omega$-dependent, and represents the solution for $P_{\mathrm{f}}=-1$. Eq. (46) can then be written as follows in Fourier space:

$$
K_{\mathrm{f}} \Delta P_{\mathrm{f}}=\left[\phi^{\prime} C^{*}+\phi\left(1-\phi^{\prime}\right) C^{*}-\phi C^{*}\langle k\rangle_{\mathrm{eff}}\right] \mathrm{i} \omega P_{\mathrm{f}}
$$

with

$$
\langle k\rangle_{\mathrm{eff}}=\frac{1}{\left.\mid \Omega^{\prime}\right\rceil} \int_{\Omega_{\mathrm{sp}}^{\prime}} k \mathrm{~d} \Omega
$$


Taking the inverse Fourier transform of Eq. (47) we obtain the description for a transient excitation:

$$
K_{\mathrm{f}} \Delta P_{\mathrm{f}}=\left[\phi^{\prime} C^{*}+\phi(1-\phi) C^{*}\right] \frac{\partial P_{\mathrm{f}}}{\partial t}-\phi C^{*} \int_{-\infty}^{t} \hat{K}(t-\tau) \frac{\partial^{2} P_{\mathrm{f}}}{\partial t^{2}} \mathrm{~d} \tau
$$

where $\hat{K}(t)$ is the inverse Fourier transform of $\langle k\rangle_{\mathrm{eff}} / \mathrm{i} \omega$ and characterizes the memory effects induced by the double-porosity structure of the medium. The convolution product in Eq. (48) corresponds to the phenomenological interporosity flow. The memory function $\hat{K}(t)$ is similar to the memory function introduced in Auriault (1983) and used again in Auriault and Royer (1993a), for transient heat transfer in double-conductivity composites.

The memory function $\hat{K}$ can be represented by an infinite sum of exponential terms:

$$
\hat{K}(t)=\sum_{p=1}^{\infty} a_{\mathrm{p}} \exp \left(-b_{\mathrm{p}} t\right), \quad b_{1}<b_{2}<\ldots
$$

The long-time behavior can therefore be approximated by:

$$
\hat{K}(t) \approx a_{1} \exp \left(-b_{1} t\right)
$$

With this approximation, Eq. (47) expressed in Fourier space becomes:

$$
K_{\mathrm{f}} \Delta P_{\mathrm{f}}=C^{*}\left[\phi^{\prime}+\phi\left(1-\phi^{\prime}\right)-\phi \frac{a_{1} \mathrm{i} \omega}{b_{1}+\mathrm{i} \omega}\right] \mathrm{i} \omega P_{\mathrm{f}}
$$

\subsection{Comparison between Warren and Root and homogenization models}

A similar investigation is conducted in Auriault and Royer (1993a) for heat transfer in composites with double conductivity. The reader is referred to this paper for details.

Let us now compare the two descriptions (44) and (49) in Fourier space. This comparison yields to the following identification:

$$
\frac{\phi C^{*} s K_{\mathrm{p}}}{s K_{\mathrm{p}}+\phi C^{*} \mathrm{i} \omega} \equiv \phi C^{*}\left(1-\phi^{\prime}-\frac{a_{1} \mathrm{i} \omega}{b_{1}+\mathrm{i} \omega}\right)
$$

which is impossible for all values of $\omega$. Thus, the Warren and Root model cannot be identified to the long-time approximation of the homogenization model. Therefore, the Warren and Root model gives a rough description for transient excitations.

Now let us improve the interporosity flow to make the Warren and Root model suitable for the short-time behaviors. Successive integration by parts of the integral:

$$
\int_{-\infty}^{t} \hat{K}(t-\tau) \frac{\partial^{2} P_{\mathrm{f}}}{\partial t^{2}} \mathrm{~d} \tau
$$

in Eq. (48) gives an infinite sum of time-derivatives of $P_{\mathrm{f}}$. Therefore, the model for transient excitations can be written as:

$$
K_{\mathrm{f}} \Delta P_{\mathrm{f}}=\left[\phi^{\prime} C^{*}+\phi\left(1-\phi^{\prime}\right) C^{*}\right] \frac{\partial P_{\mathrm{f}}}{\partial t}-d_{2} \frac{\partial^{2} P_{\mathrm{f}}}{\partial t^{2}}-\ldots d_{n} \frac{\partial^{n} P_{\mathrm{f}}}{\partial t^{n}}-\ldots
$$

Eq. (51) suggests that we must correct the term of interporosity by introducing a time derivative of $P_{\mathrm{f}}$ :

$$
q=s K_{\mathrm{p}}\left(P_{\mathrm{p}}-P_{\mathrm{f}}-\theta \frac{\partial P_{\mathrm{p}}}{\partial t}\right)
$$

where $\theta$ is a constant. 
It can be shown (Auriault and Royer, 1993b; Royer, 1994) that with this correction, the Warren and Root model can then be identified with the long-time approximation of the homogenization model. The correction (52) is similar to those used in a recent work (Bai et al., 1994), where the derived analytical solution is compared to the analytical solution of the complete Barenblatt model.

\section{Conclusions}

Since the first mathematical double-porosity model has been published (Barenblatt et al., 1960), modeling transport phenomena in such media has been the subject of active research. Through the classical phenomenological approach, the problem is directly treated at the macroscopic scale. Thus, the influence of the local heterogeneities on the macroscopic behavior cannot be perfectly disclosed. Furthermore, negligible phenomena such as flow in the pores or storage capacity in the fractures are often simply discarded.

The homogenization method for periodic structures allows derivation of the macroscopic behavior from the complete microscopic description. Since there is no macroscopic prerequisite, the influence of the local effects is conveyed to the macroscopic level. The present results show that the notion of uniqueness is meaningless for the double-porosity media model. Indeed, the relationship between the separations of scales states the macroscopic behavior. The three different cases of scale separation lead to three different macroscopic behaviors. In both investigations, the maximum coupling effect is obtained for equally separated scales. The results in Section 3 constitute the first models of gas flow through a double-porosity medium that takes the strong compressibility of the fluid into account. As for the second investigation, it shows a remarkable characteristic conceming consolidation of double-porosity media: the asymmetry of the coupling tensors. For a slightly compressible fluid, pseudo-steady-state phenomenological approaches are shown to be rough approximations for transient excitations. Then, the phenomenological models are improved by introducing a fracture pressure time-derivative term in the interporosity flow.

\section{References}

Auriault, J.L., 1983. Effective macroscopic description for heat conduction in periodic composites. Int. J. Heat Mass Transfer, 26(6): $861-869$.

Auriault, J.L., 1991. Heterogeneous medium - Is an equivalent macroscopic description possible? Int. J. Eng. Sci., 29(7): 785-795.

Auriault, J.L. and Boutin, C., 1992. Deformable porous media with double porosity — Quasi-statics, I: Coupling effects. Transport Porous Media, 7: 63-82.

Auriault, J.L. and Boutin, C., 1993. Deformable porous media with double porosity — Quasi-statics, II: Memory effects. Transport Porous Media, 10: 153-169.

Auriault, J.L. and Royer, P., 1993a. Double conductivity media: A comparison between phenomenological and homogenization approaches. Int. J. Heat Mass Transfer, 36(10): 2613-2621.

Auriault, J.L. and Royer, P., 1993b. Écoulement d'un gaz dans un milieu poreux à double porosité. C.R. Acad. Sci. Paris, Sér. II, pp. 431-436 (abridged English version).

Auriault, J.l., Strzelecki, T., Bauer, J. and He, S., 1990. Porous deformable media saturated by a very compressible fluid: Quasi-statics. Eur. J. Mech. A/Solids, 9(4): 373-392.

Bai, M., Roegiers, J.C. and Elsworth, D., 1994. An alternative model of fractured reservoir simulation. In: Siriwardane and Zaman (Editors), Computer Methods and Advances in Geomechanics. Balkema, Rotterdam, pp. 2031-2035.

Barenblatt, G.I., 1963. On certain boundary value problems for the equations of seepage of liquid in fissured rocks. PMM, 27(2): $348-350$.

Barenblatt, G.I, Zheltov, I.P. and Kochina, I.N., 1960. Basic concepts in the theory of seepage of homogeneous liquids in fissured rocks. PPM, 24(5): 852-864.

Barenblatt, G.I., Entov, V.M. and Ryzhik, V.M., 1990. Theory of Fluid Flows through Natural Rocks. Kluwer, Dordrecht.

Biot, M.A., 1941. General theory of three-dimensional consolidation. J. Appl. Phys., 12: 155-164.

Boutin, C., 1994. Comportement macroscopique de matériaux hétérogènes. Thèse d'Habilitation, Université Joseph Fourier, Grenoble.

Chen, Z.X., 1989. Transient flow of slightly compressible fluids through double-porosity, double-permeability systems — A state of the art review. Transport Porous Media, 4: 147-184. 
Chen, Z.X., 1990. Analytical solutions for the double-porosity, double-permeability and layered systems. J. Pet. Sci. Eng., 5: 1-24.

Gringarten, A.C., 1984. Interpretation of tests in fissured and multilayered reservoirs with double porosity behavior: Theory and practice. J. Pet. Technol., Distinguished Authors Ser., 4: 549-564.

Levy, T. and Sanchez-Palencia, E., 1975. On boundary conditions for fluid flow in porous media. Int. J. Eng. Sci., 13: $923-940$.

Royer, P., 1994. Contribution de l'homogénéisation a l'étude de la filtration d'un gaz en milieu déformable a double porosité - Application à l'étude du système gaz-charbon. Thèse, Université Joseph Fourier, Grenoble.

Royer, P. and Auriault, J.L., 1992. Very compressible fluid flow through a porous rigid medium with double porosity. Stud. Geotech. Mech., 13(1/2): 65-77.

Royer, P. and Auriault, J.L., 1994. Transient quasi-static flow through a rigid porous medium with double porosity. Transport Porous Media, 17(33): 33-57.

Warren, J.R. and Root, P.J., 1963. The Behavior of naturally fractured reservoirs. Soc. Pet. Eng. J. (Sept.), pp. $245-255$. 